CAHIERS DE

NARRATOLOGIE

\section{Cahiers de Narratologie}

Analyse et théorie narratives

$30 \mid 2016$

Street Art 2

\title{
Interventions in situ à Lisbonne : étude des cas d'Os Gémeos, de Vhils, de Bicicleta sem Freio et de Nunca
}

Addressing street art murals as site-specific interventions in Lisbon: Case-

studies of Os gémeos, Vihls, Bicicleta sem Freio and Nunca

\section{Helena Elias et Inês Marques}

\section{OpenEdition}

\section{Journals}

Electronic version

URL: http://journals.openedition.org/narratologie/7515

DOI: 10.4000/narratologie.7515

ISSN: 1765-307X

\section{Publisher}

LIRCES

\section{Electronic reference}

Helena Elias et Inês Marques, «Interventions in situ à Lisbonne : étude des cas d'Os Gémeos, de Vhils, de Bicicleta sem Freio et de Nunca », Cahiers de Narratologie [Online], 30 | 2016, Online since 28 July 2016, connection on 21 April 2019. URL : http://journals.openedition.org/narratologie/7515 ; DOI 10.4000/narratologie.7515

This text was automatically generated on 21 April 2019

Article L.111-1 du Code de la propriété intellectuelle. 


\title{
Interventions in situ à Lisbonne : étude des cas d'Os Gémeos, de Vhils, de Bicicleta sem Freio et de Nunca
}

\author{
Addressing street art murals as site-specific interventions in Lisbon: Case- \\ studies of Os gémeos, Vihls, Bicicleta sem Freio and Nunca
}

Helena Elias et Inês Marques

\section{Introduction : cartographie de la recherche portant sur les œuvres murales contemporaines}

1 Les œuvres murales inondent le quotidien de la culture urbaine contemporaine. Lorsque l'on inscrit art urbain, art public, street art ou art mural dans les moteurs de recherches, les résultats affichent principalement des fresques. Les artistes créent des œuvres in situ en s'appropriant les éléments architecturaux pour en faire des ready-made urbains et en interagissant avec la signification intertextuelle des lieux (Elias et al 2013, Elias 2015). En cela, la ville devient un palimpseste vivant d'images partagées localement et au niveau mondial via les réseaux sociaux. Par son exposition au grand public, la liberté de prise de vue, la facilité de partage et de publication en ligne, l'œuvre murale constitue un format idéal pour la culture numérique.

2 La créativité de la ville, telle que définie par l'ère du web 3.0 et ses outils de géoréférencement, a considérablement changé la façon dont l'espace public est désormais perçu, partagé et archivé. En réalité, ces nouveaux types d'œuvres, d'affichages et de manières d'attirer le regard interagissent avec l'espace public tout en le redéfinissant.

Dans Imagining the City: The Difference that Art Makes, Judith Vega commente l'interaction entre l'art et la ville et souligne la participation des médias au nouveau regard porté sur les espaces urbains. On peut observer plusieurs interventions artistiques dont les modes d'interaction ont pour origine les récentes activités culturelles des médias numériques. Qu'elles restent visibles seulement deux jours ou six mois, les fresques constituent le 
format privilégié pour alimenter les réseaux sociaux. L'affichage d'une œuvre murale est largement compatible avec celui d'un écran d'ordinateur pour une consultation en ligne ou sur d'autres appareils. La prise de vue ne nécessite aucune compétence spécifique en termes de cadrage contrairement à la photographie de sculptures où il est essentiel de prendre en compte la multiplicité des plans. Le format plat de la fresque est idéal pour un affichage sur écran.

4 Se trouve-t-on alors face à un changement de paradigme de l'art public? Ce questionnement est né des recherches sur les relations culturelles entre le Brésil et le Portugal, traditionnellement représentées à travers la commande de statues et de monuments. Nous partons du principe que notre intérêt académique pour l'art public, l'art urbain ou le street art est conditionné par la définition de l'espace propre au champ de recherche d'origine. Nous avons mis en évidence un abandon du modèle d'un espace urbain ordonné par des œuvres en trois dimensions telles que les statues. L'analyse des données relatives aux œuvres commandées et créées dans le cadre des relations portugobrésiliennes ont révélé la prédominance du format d'œuvres murales urbaines. Ainsi, cette étude portera sur le travail d'Os Gémeos, de Vhils, de Bicicleta sem Freio et de Nunca, en tant qu'études de cas d'interventions murales in situ comprises dans un tel cadre. Il faut néanmoins noter que la résurgence et la multiplication de fresques ne se limite pas à notre champ d'étude en ce qu'un nombre considérable de festivals et d'autres événements organisés partout dans le monde stimulent l'apparition de telles manifestations artistiques.

Les fresques murales urbaines ont alimenté directement ou indirectement différents secteurs économiques dominants à l'échelle locale et internationale dans de nombreuses villes. Autrefois lieux privilégiés pour exposer les créations publiques, les places et les carrefours sont désormais moins attrayants que les murs et les espaces laissés disponibles après planification urbaine. Pourtant, il semble qu'en dépit de la nouvelle attractivité du format mural établie par notre recherche empirique, les fresques contemporaines sont encore peu considérées par la recherche artistique académique. Dès lors que les travaux de ces artistes sont disséminés partout dans le monde et reconnus grâce à certaines singularités (personnages récurrents, gamme de couleurs, sujets similaires), notre rôle sera de comprendre comment ces œuvres entrent en contact avec les lieux, proposent une approche in situ des espaces publics et répondent au cadre des relations interculturelles entre le Brésil et le Portugal.

6 Outre la spécificité du contexte culturel, notre étude tentera de répondre à des questions précédemment posées par le domaine de recherche en arts, en ce qui concerne particulièrement les singularités de ces travaux au niveau mondial: la résurgence de l'expression murale en tant que phénomène planétaire, la spécificité de leurs styles respectifs permettant d'identifier des artistes au niveau mondial, la communication des work in progress sur Internet et la prise en compte du geste artistique en tant que performance. La définition des pratiques et des travaux in situ établie par Kwon et Kaye est celle que nous retenons principalement pour notre évaluation de ce même aspect dans les fresques contemporaines. En prenant en compte la dispersion des œuvres au niveau mondial, notre recherche cherchera à comprendre la relation entre les singularités des fresques permettant d'identifier les artistes et la manière dont elles engagent un dialogue avec l'environnement historique, politique et économique de Lisbonne. Nous prenons également en considération les interventions urbaines de mouvements surréalistes et dada en tant qu'annonciatrices des positions contemporaines vis-à-vis de l'espace public. 
La raison pour laquelle nous intégrons les pratiques urbaines modernistes est liée au nouveau regard qu'elles ont porté sur la cité et leur redéfinition de l'espace public. De plus, leurs stratégies d'interaction avec la ville continuent d'être appliquées par de nombreux artistes. À travers leurs approches, les dadaïstes et les surréalistes ont mis en avant le processus, la performance et la subversion. Notre recherche s'appuiera aussi sur une méthodologie visuelle et se centrera sur l'interaction avec les lieux en prenant en compte la surface d'inscription, l'environnement et l'attachement symbolique des différents sites.

\section{La spécificité des œuvres in situ}

7 Un travail in situ est d'ordinaire décrit comme une œuvre spécifiquement conçue pour un endroit précis et qui entre en résonance avec celui-ci. Afin de résister à la marchandisation, l'art in situ a adopté différentes stratégies, redéfinissant ainsi le concept même d'in situ depuis son apparition. Le terme "site " désigne à la fois un réseau de différents lieux ou d'espaces mais aussi, d'un point de vue plus géographique ou architectural, un lieu de vie ou de passage (Wilkey 2004). À partir des concepts d'espace et de lieu tels que définis par De Certeau et de ceux de lieux et de non-lieux proposés par Augé, Nick Kaye propose une définition du site et de l'in situ conçu comme une extension du site en ce que cette pratique " met en place des échanges entre l'œuvre d'art et les lieux servant à sa propre signification » (Kaye 2001). Le site, sa matière, ses contours ainsi que l'ensemble des informations qu'il fournit constituent des instruments pour l'œuvre ancrée dans le lieu. L'artiste considère diverses approches qui ne concernent pas simplement l'aspect physique et réel de l'espace mais qui se conçoit comme une extension du site (Kaye 2001). Ceci peut se comprendre en termes métaphoriques ou physiques car ils renvoient à la fois à des espaces réels ou virtuels (Kaye 2001).

8 Par ailleurs, Miwon Kwon (2002) qui retrace la généalogie des pratiques in situ dans le contexte nord-américain identifie un changement de définition pratique du site passant du lieu physique à l'univers discursif et incluant désormais les espaces virtuels et immatériels. Depuis ses origines qu'elle re-situe à la fin des années 1960, alors que les premières expériences étaient principalement marquées par une compréhension empirique des lieux et liées à leur configuration physique, Kwon établit trois axes - phénoménologique, institutionnel, discursif - dont dépend le concept même de site $^{1}$ (Kwon 2002 :11-31). Plus qu'un espace physique, le terme "site» désigne, depuis ces dernières années, un processus lié à un intérêt social et politique. En tant que critiques anti-institutionnelles, les pratiques artistiques in situ se tiennent non seulement à l'écart des contextes conventionnels mais sont conçues et se développent en tant que processus défiant les règles du marché de l'art. Cette caractéristique phénoménologique de l'art in situ donne lieu à un paradigme institutionnel. Les œuvres in situ ne sont ainsi pas pérennes et relèvent d'une expérience transitoire installée dans un lieu spécifique. La dématérialisation des travaux va de pair avec la dématérialisation du site. Un troisième paradigme, relatif au discours, concerne les travaux in situ ou les projets récents liés à un espace dont l'objectif premier n'est pas tant de remettre en cause les institutions artistiques mais d'élargir leur rayonnement public en s'adaptant aux parcours nomades et aux intentions créatives des artistes. Les pratiques in situ actuelles ne dépendent pas d'un lieu particulier comme condition première. À l'inverse, le site est choisi en fonction des projets poursuivis par les artistes. D’après Kwon (2002:47), « il découle du travail lui- 
même (souvent en tant que contenu) et sa convergence avec les formations discursives préexistantes est ensuite vérifiée ${ }^{2} »$.

9 En tant que lieu physique et domaine social, le site est subordonné à une formation discursive désignée par le champ conceptuel, l'échange intellectuel et le débat culturel. Eclairées par une large gamme de disciplines provenant des sciences sociales et tournées vers la culturelle populaire, les pratiques contemporaines in situ se caractérisent par une prédominance discursive. Par ailleurs, le flux constant d'artistes qui se déplacent à l'international afin de réaliser des projets dans différentes villes en réponse aux exigences d'un réseau artistiquement mondialisé est incompatible avec une certaine pérennité des travaux et un engagement au long terme sur le site. Ainsi, le site se comprend désormais dans un champ d'opération discursif, sa localisation est multiple et il opère à travers une logique intertextuelle. Même s'ils sont apparus l'un à la suite de l'autre, ces trois paradigmes existent à présent simultanément dans les pratiques in situ contemporaines. Pourtant, on observe une véritable tendance à se distancier du lieu concret. Ayant redouté ce changement, Kwon déplore le recul d'une certaine permanence, d'une continuité et d'un ancrage dans les travaux in situ :

Non seulement l'œuvre d'art tend à perdre son lien physique avec le lieu, mais le sujet choisi par l'artiste s'est également libéré de toute engagement pérenne vis-àvis des circonstances locales. Les qualités de permanence, de continuité, d'affirmation et d'ancrage (qu'il soit physique ou autre) semblent d'un autre temps et donc politiquement suspectes dans un tel contexte. À l'inverse, l'instabilité, l'ambiguïté et la fugacité sont perçues comme des attributs positifs marquant l'aspect progressiste du travail artistique ${ }^{3}$.

10 Les travaux d'Os Gémeos, de Vhils, de Bicicleta sem Freio et de Nunca pris en tant qu'étude de cas des interventions murales in situ et représentatifs des tendances actuelles en matière d'art public sponsorisé correspondent au troisième paradigme à bien des égards. Limiter les fresques urbaines au concept d'art in situ s'avère être une tâche complexe. La notion d'art in situ a été abordée dans le cadre du street art mais elle n'a pas fait l'objet d'une réflexion plus précise en ce qui concerne les interventions murales (Kuttner 2014). Dans Walls of Empowerment, Latorre associe le concept d'environnement mural à l'in situ puisqu'il a pour origine l'emplacement et l'espace physique de la fresque (Latorre 2008). En effet, la fresque installée sur le mur crée un réseau de relations avec l'espace environnant.

\section{Les précurseurs : les ready-made urbains et les monuments improvisés}

Les pratiques spatiales des groupes dada et surréalistes peuvent être considérées comme annonciatrices des interventions sur site en milieu urbain. Le parcours dada à l'église St Julien-le Pauvre (1921) et la réponse surréaliste à la statuophobie peuvent se classer dans une définition plus large de l'œuvre in situ. Le parcours dada relève du ready-made urbain et peut se comprendre comme une déclaration faite dans et pour l'espace public en ce que cette excursion était ponctuée de moments d'interaction avec les passants. De multiples dispositifs ont été mis en place: la lecture d'un manifeste ou de définitions du dictionnaire Larousse prises au hasard comme explications aux sculptures ou aux colonnes de la cour de l'église et la parodie d'une visite guidée. À travers le processus mimétique visant à considérer les visites guidées de Paris comme un sujet de dérision, le 
groupe dadaïste a offert une opération de réassignation des espaces peu fréquentés. Ce faisant, ces artistes ont attribué une valeur esthétique à l'espace vide, un endroit qui, jusqu'alors, n'avait pas lieu d'exister. Cette première excursion dada marque l'avènement d'une esthétique de l'action, créatrice d'un événement visible par tous dans l'espace public citadin. La nature essentiellement performative de l'événement conçu pour établir un contact et agir avec le public a rencontré une adhésion inattendue, allant même jusqu'à étonner les artistes eux-mêmes (Bishop 2012).

12 La ville de Paris a également été le terrain d'une approche critique à travers la photographie et la littérature mise en place, cette fois, par les surréalistes. Ces derniers tournent leur attention vers les monuments et les statues exposés dans les jardins ou les grandes places. À cette époque, le nombre excessif de statues ou de bustes du XIXe siècle disséminés à travers la ville - une pratique désignée comme le phénomène de statuomani ${ }^{4}$ - commençait à être remis en cause par les autorités locales qui s'interrogeaient à propos de leur permanence dans l'espace public. Ces formes sculptées sont alors devenues l'une des préoccupations surréalistes, comme en attestent les travaux de Louis Aragon, d'André Breton, de Paul Éluard ou de Robert Desnos ${ }^{5}$ (Baker 2007). Mais au lieu de critiquer cette statuomanie, certains surréalistes tels que Desnos ou Boiffard ont émis l'idée que les statues possédaient des qualités intrinsèques dignes d'être révélées. Le magazine Documents fournissait des informations relatives aux sculptures et à d'autres créations et contribuait à nier une hiérarchie entre l'art et d'autres formes primitives (Levaillant 2012). Baker décrit le texte de Robert Desnos Pygmalion et le Sphinx comme le constat de l'échec de la sculpture et des statues dans leur volonté de dépeindre les portraits célèbres de la nation telles des figures immortelles. La matérialité du marbre et du bronze soulignait plus le poids des statues que la silhouette des personnages idéalisés. Les tentatives des sculpteurs pour contourner ces difficultés de représentation semblaient illogiques. En dépit du piédestal surélevant les figures, des ballons de bronze, des nuages de pierre et des veines de marbre, les sculptures demeuraient piégées par la gravité, ce qui révélait leur matérialité essentielle. Un tel sujet a incité les photographes à mettre en scène des interventions ready-made sous forme de monuments improvisés. Le photographe Jacques-André Boiffard est l'auteur de cinq photographies de monuments en bronze accompagnées du texte de Desnos. Partageant le point de vue de l'auteur, Boiffard a sélectionné des angles de vue qui soulignent la vérité matérielle des statues par la mise en relief de certains éléments (Baker 2007). Par exemple, pour la statue de Louis IV, le bronze semble fondre à l'arrière-plan tandis que sur les photos de monuments en marbre, la pierre blanche s'efface sous la lumière du jour. En explorant les espaces urbains à travers des visites guidées, des parcours, des écrits et d'autres ressources, les dadaïstes et les surréalistes ont porté une nouvelle lumière sur l'acte d'évoluer dans l'espace public. Ils ont élevé l'inscription du mouvement dans l'espace et le temps au rang d'opération esthétique. En ce qui concerne la spécificité in situ, ils ont exploré de nouvelles formes d'engagement et d'interaction avec l'environnement en développant des stratégies pour dynamiser la ville à travers, par exemple, la visite d'endroits précis conçus comme des ready-made ou en mettant en scène des déambulations afin de stimuler la découverte de la ville (Carreri 2001). 


\section{Point de contact entre notre approche et les fresques au niveau mondial}

13 Vers la fin du XIXe siècle, les espaces publics parisiens de type Haussmannien se caractérisaient par la présence de statues et de monuments. Cette esthétique urbaine a servi de modèle pour nombre de capitales européennes qui ont établi à leur tour la sculpture comme médium prédominant dans l'espace public. Mais le XXe siècle a marqué le déclin progressif de la sculpture, et plus précisément des statues, à la faveur d'autres expressions artistiques en milieu urbain ${ }^{6}$. Dans certains contextes culturels, la production de statues et de bustes a néanmoins été maintenue (Elias 2007). C'est le cas des relations culturelles $^{7}$ entre le Portugal et le Brésil au XXe siècle, qui ont commémoré les événements historiques des deux pays essentiellement à travers la production de statues et de monuments (Elias et al 2013). Au Portugal, après la révolution des œillets (1974), émergent différentes formes artistiques dans l'espace public, menant ainsi à une certaine diversité de représentations (image 1) même si la plupart des œuvres commandées sont encore des sculptures. Le début du XXIe siècle marque un tournant dans les sujets, les discours et les modes de représentation, qui se détachent des rituels commémoratifs apparus au XIXe siècle. D'autres pratiques d'art public ont émergé grâce au soutien des partenaires et des chargés de projets favorisant de nouveaux médias et langages visuels, une plus grande diversité de sujets ainsi que l'implication d'autres agents. Les fresques urbaines sont désormais privilégiées pour promouvoir les relations culturelles; elles présentent ainsi les langages visuels d'une jeune génération d'artistes opérant au niveau mondial et dont les sources sont ancrées dans le graffiti et le street art (Elias 2015).

Les travaux d'Os Gémeos, de Vihls, de Bicicleta sem Freio et de Nunca, invités par la galerie Underdogs à exposer les œuvres à Lisbonne, entrent dans ce cadre. Le soutien de la galerie d'art urbain administrée par la ville a permis à Lisbonne d'établir sa réputation de ville tournée vers les créations en milieu urbain. Les réalisations ont également permis de placer les relations portugo-brésiliennes dans un contexte plus large à travers une communication mondiale, signalant un changement radical vers une plus grande créativité liée à la ville. Ces œuvres sont aussi liées aux récents changements dans les procédures de commandes et de production de travaux in situ à travers le monde. Invités par diverses institutions artistiques dans un réseau d'art toujours plus mondialisé, les artistes se déplacent constamment et travaillent souvent sur plusieurs projets in situ à la fois. Leurs réalisations dépendent donc plus de leurs projets artistiques et, sans nécessairement recourir à un ancrage pérenne sur les sites, elles se construisent à travers une logique intertextuelle. Une recension de l'in situ doit donc prendre en considération la manière dont l'ancrage avec le lieu est compensé par le rayonnement de l'artiste dont l'identification constitue un élément capital (Kwon). Cet «aspect performatif du mode opératoire de l'artiste", tel que le nomme Kwon, s'avère être la principale qualité artistique qui est ensuite relayée sur le réseau d'art mondial. Ainsi, l'artiste devient le garant de l'authenticité, de la répétition et de la circulation de l'œuvre. 


\section{Les travaux d'Os Gémeos, de VHILS, de Bicicleta sem Freio et de Nunca}

\section{Os Gémeos}

15 L'intervention d'Os Gémeos à Lisbonne résulte du soutien de l'association Azafama Citadina, de la Gallery of Urban Art (GAU) et du conseil municipal de Lisbonne (CML). La GAU a été créée pendant les années 2010-11 et son principal objectif consistait alors en la réalisation de projets street art et de graffiti à différents endroits dans la ville afin de promouvoir l'art visible pour tous dans l'espace urbain. D'autres institutions, à la fois privées et publiques, ainsi que des associations se sont impliquées dans des commandes d'œuvres. Une fois la proposition mise en forme, le conseil municipal de Lisbonne a rejoint l'initiative et a apporté son soutien officiel aux interventions (Neves and Lopes 2015).

16 Caractérisées par des personnages jaunes peints sur des murs bruts, les œuvres d'Os Gémeos présentes à travers le monde établissent différents degrés d'interaction avec l'environnement. En général, les personnages semblent émerger des murs telles des figures érigées sur un fond brut souvent monochrome. Les artistes brésiliens d'Os Gémeos sont réputés pour les messages politiques et sociaux de leurs œuvres murales où l'on retrouve les bonhommes jaunes qui sont la signature du duo à travers le monde. Os Gémeos n'avaient pas de projet précis de sujet ni de demandes spéciales pour le travail à Lisbonne. Ils ont d'abord tracé leur personnage jaune typique auquel ils ont rajouté un bandeau dissimulant en partie le visage. Un détail du bandeau doit être remarqué : au lieu de la marque, on peut lire "J'adore le vandalisme » (image 2). Peinte dans la même rue, une fresque de Blu affiche un message politique similaire en mettant en scène un homme corpulent coiffé d'une couronne dont les ornements sont les logos de multinationales pétrolières. Ces travaux font usage de l'intertextualité au niveau local et mondial et dépassent la reconnaissance des personnages dessinés de Blu ou d'Os Gémeos dans diverses grandes métropoles mondiales. Les œuvres sont situées sur l'une des artères les plus passantes qui relient deux espaces d'affaires et de commerce, Marquês do Pombal et Saldanha. Du fait de cet emplacement, les fresques s'inscrivent d'autant plus dans un contexte in situ qu'elles abordent la question du capitalisme et de la mondialisation. La taille monumentale des œuvres assure leur visibilité et l'exploitation de la jonction Saldanha/Marques de Pombal intègre cette redéfinition de l'expérience esthétique en milieu urbain. Jouissant de la même dimension imposante que les autres monuments présents dans le quartier, ces fresques remettent en question les autorités économiques internationales en place dans la ville (Elias 2015). Ces deux interventions adoptent une posture critique à l'égard des pouvoirs économiques, du capitalisme et de la mondialisation. Dans la fresque d'Os Gémeos, le personnage jaune émerge du mur et dirige son lance-pierre vers l'avenue Fontes Pereira de Melo, un boulevard reliant les deux grandes artères lisboètes.

\section{Vhils}

Directeur de la galerie Underdogs à Lisbonne, Vhils fait ressurgir le passé des lieux dans lesquels il intervient. Par le procédé de gravure qu'il utilise, l'artiste attire l'attention sur 
les différentes strates d'espace public et sur son histoire. Dans le cadre de l'année du Portugal au Brésil, Vhils a exposé son travail à la galerie Clark et a collaboré avec les communautés locales de Ladeira dos Tabajaras et de Botafogo à Rio de Janeiro en 2013. Vhils et son équipe ont réalisé un reportage pendant quatre semaines sur la démolition des bâtiments du quartier et ont gravé les portraits de certains habitants sur les murs de leurs anciennes résidences. Les immeubles ont été démolis pour le projet de reconstruction dans le cadre de la Coupe du monde de football organisé au Brésil en 2014. Le travail incorpore en arrière-plan les façades d'origine.

\section{$B S F$}

Bicicleta sem Freio (BSF) est un duo de designers et illustrateurs réputés mondialement pour leurs compositions colorées mettant en scène des femmes dans une ambiance pop années 70. Les fresques du collectif sont régies par un grand dynamisme qui est rendu par les longues chevelures et d'autres éléments qui s'étirent dans de multiples directions, répétant ainsi le mouvement des lignes de composition. Les fresques emplissent l'intégralité de façades des bâtiments tels des écrans; leurs formes sont ainsi subordonnées à celles du bâtiment.

L'intervention réalisée par BSF à Lisbonne sur Cais do Sodré entre dans ce même cadre. Le travail est le fruit d'un appel lancé par la galerie Underdogs à BSF pour la réalisation d'une œuvre publique à Lisbonne en 2012. Reprenant le thème de la mer, le travail de BSF consiste en une composition ondulante structurée par des éléments marins submergeant une jeune femme. La composition souligne l'importance du mouvement de la vague et chaque façade peut être perçue comme un plan différent dans la progression du courant en direction de Lisbonne. Continuité, répétition et séquence sont des notions-clés pour comprendre l'œuvre murale. La composition tire avantage de la similitude des trois murs sur laquelle elle s'étend. Les éléments architecturaux forment des encadrements blancs qui découpent le dessin en différents plans afin de rendre la composition pareille à une bande dessinée ou à un triptyque. Pour le passant ou le passager d'un train, un certain degré de continuité est mis en évidence par les couleurs de la mer, du ciel ou du bateau. La vue de la fresque rappelle au spectateur diverses influences. Depuis l'inspiration pop jusqu'aux filles des illustrations commerciales peintes à la main pour de l'huile solaire, en passant par les femmes des œuvres de Lichtenstein ou le personnage de Barbarella, les figures féminines rappellent aussi les modèles classiques telles que la Vénus de Botticelli ou la Méduse du Caravage ou de Bernini.

Il est impossible de nier la relation entre le passé du lieu et les éléments de la fresque qui rappellent l'histoire de Lisbonne et utilisent un imaginaire commun à la ville. Les mots Clube Naval de Lisboa font un lien avec la précédente activité du bâtiment et donnent du sens à l'œuvre en établissant un contact entre le dessin et le mur. Les termes permettent également d'indiquer la direction à suivre dans la composition. Club Naval Lisboa sont les trois seuls mots qui explicitent et permettent de lire l'image. Ces éléments font référence à l'action du bateau et aux vagues encerclant le corps de la jeune femme. Lisbonne figure au-dessus de son visage comme s'il s'agissait de son prénom. Une telle lecture fait apparaitre l'œuvre tel un portait de Lisbonne renouant avec son passé marin et ses récits tragiques de naufrages. D'autres connections sont envisageables, notamment l'évocation du tsunami qui détruisit Lisbonne en 1755 ou encore l'allégorie de la ville sous les traits d'une femme peinte dans les portraits portugais du XIXe siècle. 


\section{Nunca}

21 par rapport aux lieux où ils sont exposés. Au Brésil, l'artiste confronte le pays à son passé en dessinant des figures semblables à des brésiliens natifs (image 4). À Berlin, Nunca a créé un personnage punk tandis que près de Londres, il a représenté une abeille, symbole de la reine dont la pollinisation et la production de miel rappellent symboliquement le colonialisme britannique et l'impérialisme.

C'est la galerie Underdogs qui a invité l'artiste à créer une fresque à Lisbonne. L'œuvre est directement liée au passé historique des deux pays puisque Nunca a peint le portrait de Pedro Álvares Cabral (image 5), un navigateur portugais dont l'arrivée au Brésil en 1500 marqua le début de la colonisation du territoire par les Portugais et des outre Européens. Nunca a représenté cette figure célèbre afin d'évoquer la réalité économique et la crise financière qui touche le pays depuis ces deux dernières années. Le navigateur Pedro Á lvares Cabral, qui incarne ici le Portugal, demande l'aumône. Cabral tient dans la main une boîte de conserve et mendie comme l'indiquent les quelques euros qu'il s'apprête à recevoir. La représentation d'euros situe l'œuvre dans le temps présent et rappelle la dette du Portugal envers l'Union Européenne.

Le mélange de techniques que Nunca utilise est symboliquement chargé. La proximité avec l'univers du graffiti est visible à travers le recours au spray pour marquer les contours des formes sur le mur. Ce procédé qui fait ressortir les couleurs s'inspire des techniques graphiques du pop art. Les lignes noires qui accentuent les formes sont les caractéristiques les plus saillantes du travail de Nunca. Le recours à ces éléments graphiques peut être lié à l'histoire de la production d'images à une époque où la gravure et le dessin étaient les moyens les plus courants. L'application de lignes croisées pour créer des effets d'ombre et différents tons correspond à la technique de la hachure croisée. Très populaire aux XVe et XVIe siècles, ce procédé servait à rendre l'illusion d'une profondeur en diversifiant la quantité, l'épaisseur et l'espacement des lignes (Petherbridge 2011). L'appropriation de cette technique par Nunca pour un travail relatif au passé des populations indigènes rappelle la domination culturelle exercée par les colons qui utilisaient ce procédé pour représenter les peuples du Brésil. Les premières images trouvées dans des ouvrages du XVIe siècle employaient en effet cette technique.

\section{Remarques finales : les conditions de réalisation, les sujets et les techniques employés pour les interventions murales in situ}

Si l'on prend en compte les pratiques ayant favorisé l'espace public urbain plutôt que les espaces institutionnels et ayant ainsi considéré la ville comme l'objet de l'intervention, les ready-made urbains et les monuments improvisés annoncent les travaux in situ du XXIe siècle. Les fresques réalisées de nos jours sont définies par rapport à leur processus mis en relief par les médias qui suivent la progression et attestent de la présence de l'artiste. Face à l'incertitude quant à l'inscription pérenne des fresques, les réseaux sociaux partagent la réalisation du travail ainsi que le résultat final. Le point de départ, la performance liée à la réalisation de l'œuvre et l'information de sa progression sont des 
éléments capitaux. L'artiste travaille au niveau mondial, se déplace de ville en ville et intervient dans l'espace public. Comme le remarque Kwon, les street artists voyagent à travers le monde pour réaliser des projets in situ dans différentes villes afin de répondre à la demande d'un réseau d'art mondialisé, ce qui n'est pas compatible avec un engagement durable sur un site spécifique.

Dans cette optique, ces travaux rentrent plus dans la catégorie des pratiques discursives liées à un site car elles opèrent un lien avec le lieu selon une logique intertextuelle, comme suggéré par Kwon. L'identification des artistes, de leurs techniques et de leurs sujets résulte des similarités dans les procédés de réalisation, les couleurs et les formes représentées et ne provient pas seulement de leur engagement avec l'espace public. Ce sont là les véritables marques inscrites par les auteurs sur la surface du mur et qui permettent au spectateur, où qu'il soit, de reconnaitre l'identité de l'artiste. L'emploi systématique de ces éléments visuels relance le débat autour du culte voué à l'artiste, en ce qui concerne notamment la fascination qu'exerce la possible identification par le trait, le geste et les techniques qui ont fait, par exemple, la réputation de Jackson Pollock ou d'Yves Klein. Bien qu'ils renforcent leur notoriété et fassent grimper leur cote sur le marché international, ces artistes poursuivent leur critique du pouvoir économique en intégrant dans leurs représentations des éléments liés aux problèmes sociaux passés ou présents. Les travaux d'Os Gémeos, de Vhils, de Bicicleta sem Freio et de Nunca placent les relations culturelles entre le Brésil et le Portugal au sein d'une scène mondiale et invitent ainsi à de nouveaux types d'engagement.

Au-delà de la récurrence et de la permanence des œuvres in situ, ce qui marque désormais l'aspect ancré sur site est la manière dont les artistes répondent aux paysages urbains, en prenant en compte la préexistence du lieu et en réinventant le cadre culturel et historique servant pour représenter autrefois de tels liens. La conception de la fresque directement sur site au lieu d'une réalisation en atelier renforce aussi la relation entre l'œuvre et l'environnement. Pourtant, au sein de cette approche à la fois locale et mondiale, les travaux ne nécessitent pas forcément un haut degré de relation avec le lieu physique et sa dimension sociale dès lors que cet engagement dépend, comme nous l'avons montré, de la culture populaire intertextuelle (visuelle et matérielle). Le tableau suivant reprend les éléments essentiels de notre recherche portant sur les fresques in situ. Il indique que la manière dont un artiste choisit un lieu dans une ville est la même à travers le monde. L'ensemble des travaux sont ainsi essentiellement caractérisés par les éléments de discours, à savoir le sujet, l'interaction avec l'environnement urbain et l'approche symbolique des lieux où ils sont exposés.

\begin{tabular}{|c|c|c|c|}
\hline & Surface et contenu & Contexte urbain & $\begin{array}{l}\text { Approche symbolique et } \\
\text { interaction }\end{array}$ \\
\hline $\begin{array}{l}\text { Os } \\
\text { Gémeos }\end{array}$ & $\begin{array}{lr}\text { Façade de bâtiments } \\
\text { abandonnés. } \\
\text { Personnages jaunes } \\
\text { sortant des } & \text { murs. } \\
\text { Murs } & \text { bruts. } \\
\text { Intégration } & \text { des } \\
\text { balcons. } & \end{array}$ & $\begin{array}{l}\text { Interaction par le lance- } \\
\text { pierre visant la grande } \\
\text { avenue. Dimensions de } \\
\text { l'œuvre similaires à celles } \\
\text { des monuments alentours. }\end{array}$ & $\begin{array}{l}\text { Critique du capitalisme, de la } \\
\text { mondialisation, des pouvoirs } \\
\text { économiques } \\
\text { multinationales. }\end{array}$ \\
\hline
\end{tabular}




\begin{tabular}{|c|c|c|c|}
\hline Vhils & \begin{tabular}{ll}
\multicolumn{2}{l}{ Visages gravés sur les } \\
côtés de murs de \\
bâtiments & en \\
démolition. &
\end{tabular} & $\begin{array}{l}\text { Bâtiments abandonnés } \\
\text { intervention sur des } \\
\text { espaces en démolition en } \\
\text { attente de reconstruction. } \\
\text { Interaction avec la } \\
\text { communauté locale. }\end{array}$ & $\begin{array}{l}\text { Mise en scène de l'histoire des } \\
\text { immeubles. Portraits des } \\
\text { résidents avant la démolition. }\end{array}$ \\
\hline BSF & $\begin{array}{l}\text { Dessin couvrant les } \\
\text { trois murs à la } \\
\text { manière d'un papier } \\
\text { peint. Couleurs vives. }\end{array}$ & $\begin{array}{l}\text { Lieu en transition : lecture } \\
\text { progressive du travail } \\
\text { pour le promeneur ou le } \\
\text { voyageur en train. } \\
\text { Continuité des éléments } \\
\text { visuels. }\end{array}$ & $\begin{array}{l}\text { Représentation d'une figure } \\
\text { féminine rappelant Venus et } \\
\text { Barbarella. Détails architecturaux } \\
\text { servant d'encadrements présence } \\
\text { de mots. Allégorie de Lisbonne et } \\
\text { prise en compte de l'usage présent } \\
\text { du bâtiment. }\end{array}$ \\
\hline Nunca & $\begin{array}{l}\text { Côté d'un immeuble. } \\
\text { Figure historique. } \\
\text { Mur brut. }\end{array}$ & $\begin{array}{l}\text { Personnage posté à un } \\
\text { carrefour. }\end{array}$ & $\begin{array}{l}\text { Figure incarnant le passé } \\
\text { historique et faisant référence au } \\
\text { contexte social actuel. }\end{array}$ \\
\hline
\end{tabular}

Image 1

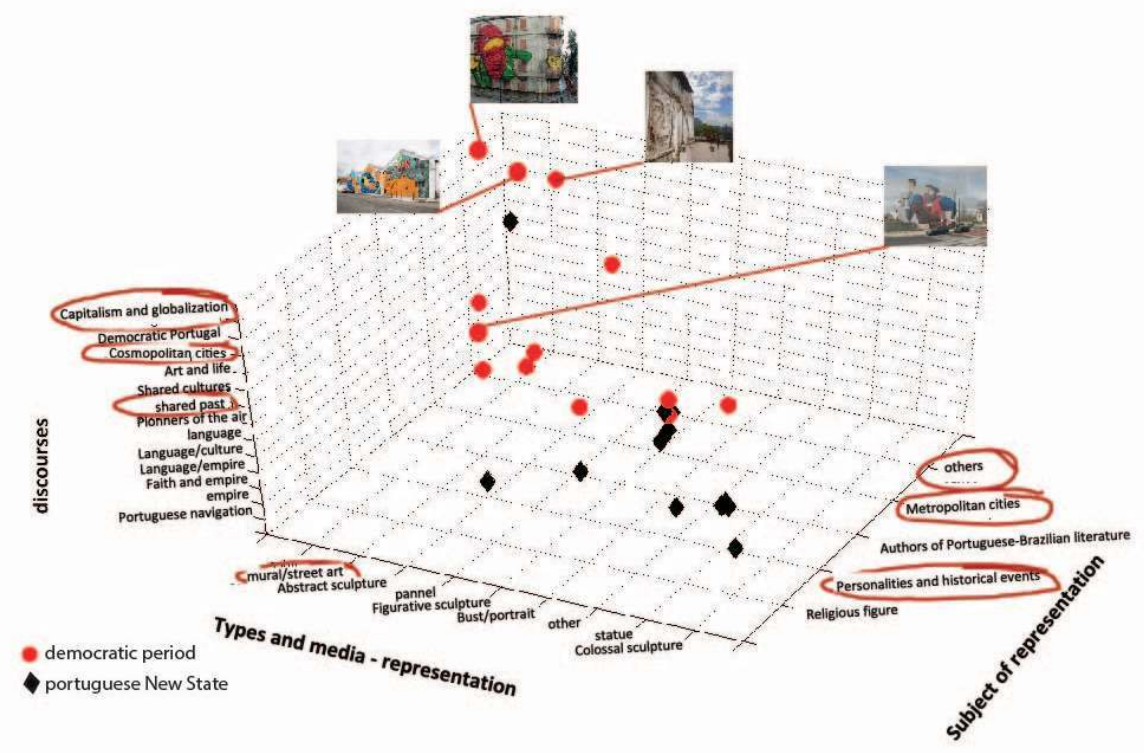

L'ART PUbLiC ART DANS LE CADRe des RELATIONS PORTUgO-BRÉSILIENNES AUTEUR : HeLENA ELIAS 
Image 2
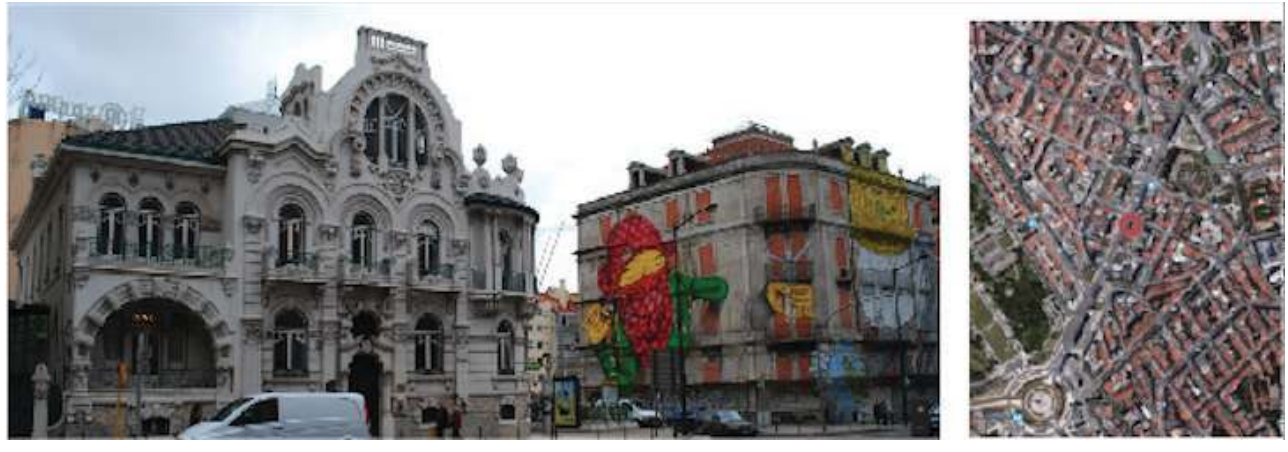

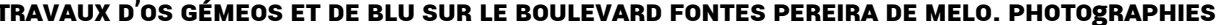
D'ELIAS, MARQUES \& LEONOR ET GOOgLE EARTH.

Image 3

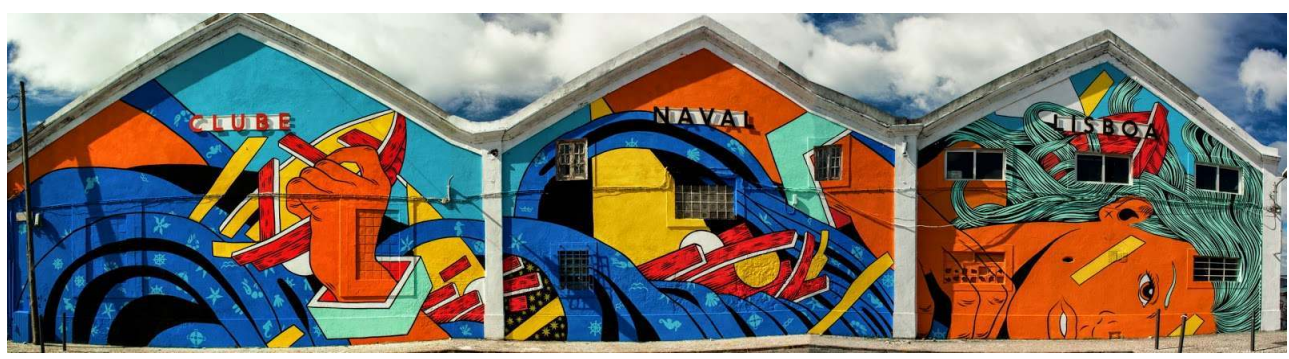

FRESQUE DE BSF AU CLUBE NAVAL DE LISBOA SOURCE : HTTPS://URBANITEWEBZINE.COM/2014/06/03/ BICICLETA-SEM-FREIO-NEW-MURAL-X-UNDERDOgS-IN-LISBON/ 
Image 4

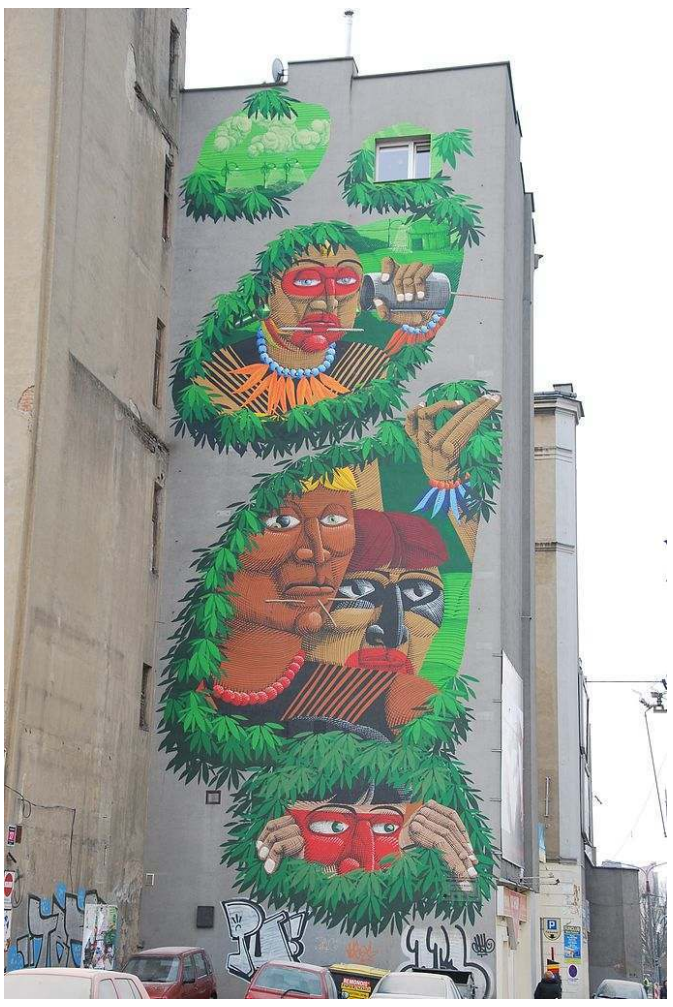

FRESQUE DE NUNCA AU BRÉSIL. SOURCE: HTTPS://COMMONS.WIKIMEDIA.ORg/WIKI/ FILE:MURAL_OF_NUNCA,_BRAZIL,_\%C5\%81\%C3\%B3D\%C5\%BA_NARUTOWICZA_KILI\%C5\% 84SKIEgO_STREETS_CORNER.JPg

Image 5

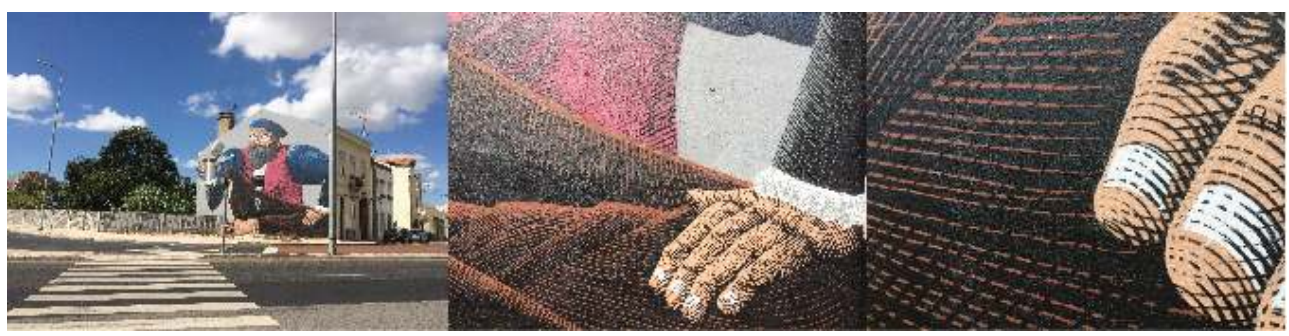

Intervention de Nunca à Lisbonne. Photographie d'Elias. 
Image 6

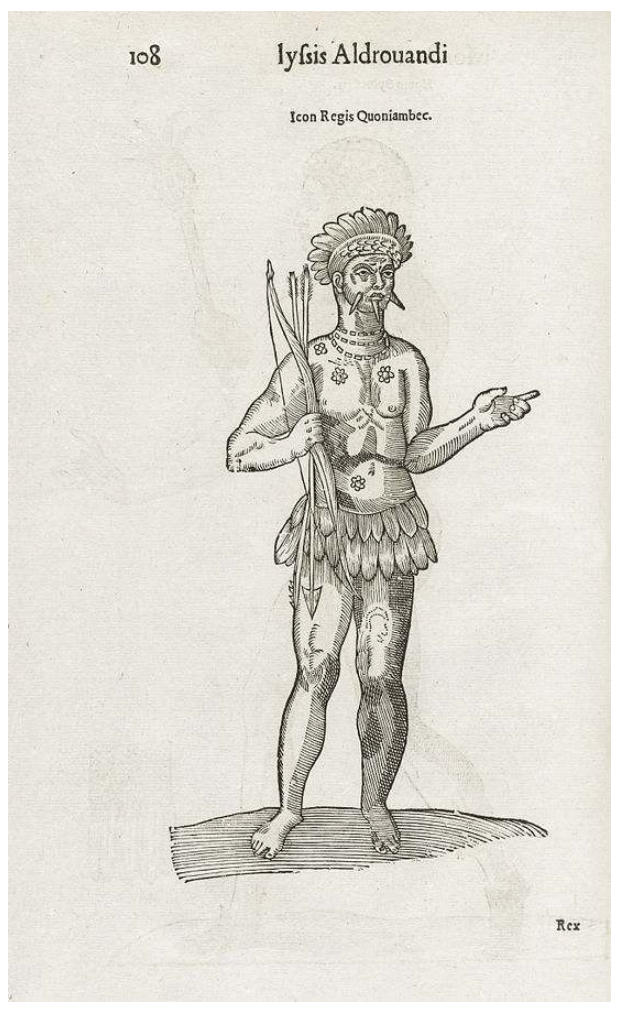

REPRÉSENTATION TRADITIONNELLE DES INDIgÈNES AU BRÉSIL. IMAgE NUMÉRÉ 51-897, HOUgHTON LIBRARY, HARVARD UNIVERSITY

Image 7

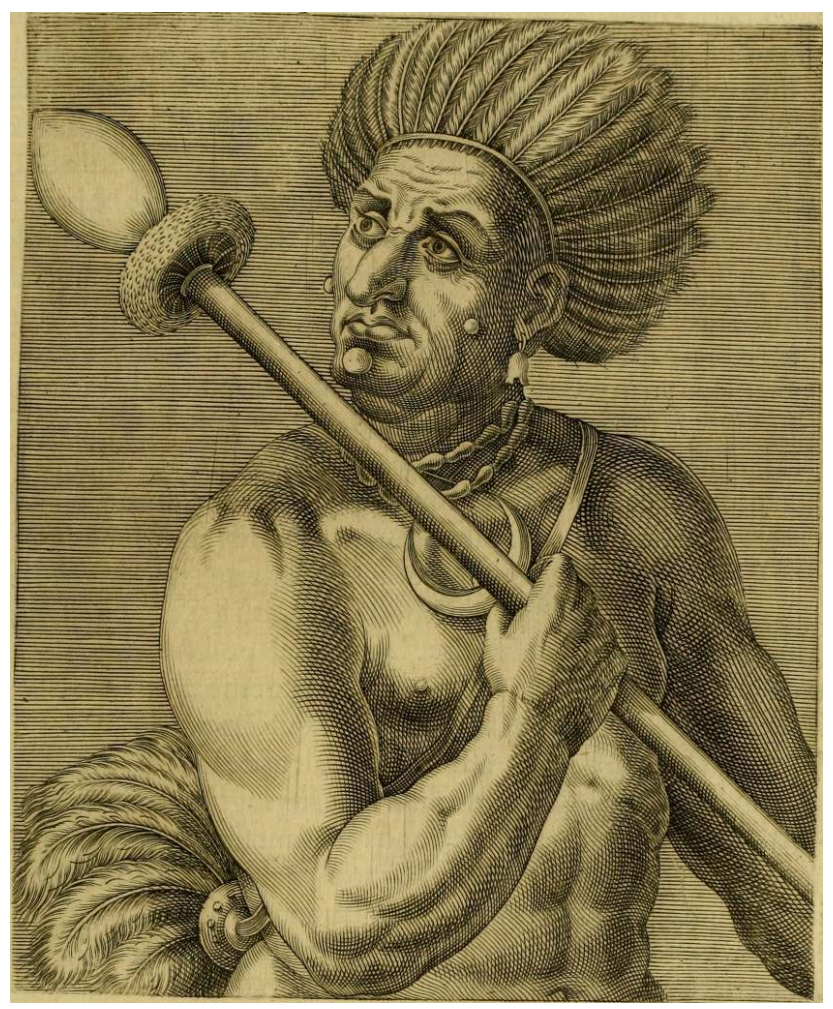




\section{BIBLIOGRAPHY}

Portrait de Cunhambebe, un chef Tupinambá qui signa le trait de paix avec les Portugais Nobrega and Anchieta. Réalisé par André Thévet - Archive en ligne, scan de Les vrais portraits et vies des hommes illustres grecs, latins et payens, domaine public.

Agulhon, M. (1988) La statuomanie et l'histoire. In : Histoire Vagabonde I: Ethnologie et politique dans la France contemporaine, Gallimard: Paris, 137-185

Abdelrahman, A. (2009) Compositional structures in mural design: Towards a sitespecific deconstructive mural methodology, $\mathrm{PhD}$ thesis, University of Bedfordshire

Baker, S. (2007) Surrealism, History and Revolution, Peter Lang: Oxford

Bouchier, M. (2006) Visites, déambulations, dérives - Paris, une ville sans coordonnées. In : Cartographies littéraire, (sous la direction de Crystel Poinçonnat et Chantal

Liaroutzos), Editions Le manuscrit : Paris, https://

territoiresthetiques.files.wordpress.com/2014/01/visites-dc3a9ambulations-dc3a9rives-parisune-ville-sans-coordonnc3a9e-2006.pdf [25.2.16]

Carreri, F. (2001) Walkscapes: Walking as an Aesthetic Practice, Gustavo Gilli : Barcelona

Elias, H. (2007) Arte Pública das Administrações Central e Local do Estado Novo em Lisboa : Sistemas de encomenda da CML e do MOPC/MOP (1938-1960). Tese de Doutoramento, Universidade de Barcelona. http://diposit.ub.edu/dspace/handle/2445/35438 [16/11/2015].

Elias, H., Marques, I., \& Leonor (2013) Public Art in the Portuguese-Brazilian cultural relationships: themes, means and discourses. (1940-2010). In : Public Art in Latin America. Santiago: Ed. Uni Santiago do Chile.

Elias, H. (2015) From squares to walls : contemporary murals of Os Gémeos, Nunca and Bicicleta sem Freio in Lisbon. In : Urban Creativity journal n. 1 vol 2 Places and non-places. Lisbon: Urban Creativity, pp. 42-49. www.urbancreativity.org/uploads/1/0/7/210727553/

journal2015_v1_n2_web_final_upt1.pdf [21-03-2016]

Hollevoet, C. (1995) Quand l'objet de l'art est la démarche - Flâneries, dérives et autres déambulations. In Exposé, n 2, p. 112-123.

Kwon, M (2002) One Place after another, Site-specific art and locational identity, Massachusetts : Massachusetts Institute of Technology

Kaye, N. (2001) Site-Specific Art, Performance, Place and Documentation, London : Routledge

Knutter, T. (2014) Os Gémeos \& São Paulo: appropriating public space in a "City of Walls", In : Lisbon Street Art \& Urban Creativity 2014 Proceedings pp.123-131. Lisbon: Urban Creativity

Latorre, G. (2008) Walls of Empowerment Chicana/o Indigenist Murals of California. Austin : University of Texas Press

Levaillant, F. (2011) Ecrire la sculpture dans « Documents », magazine illustre (1929-1930). In : Ecrire la sculpture (XIXe-XXe siècle), Paris [Online] https://halshs.archives-ouvertes.fr/ halshs-00692176

Pessard, G. (1911) Statuomanie Parisienne - Etude critique sur l'abus des statues, Paris 
Petherbridge, D. (2011) The Primacy of Drawing, Histories and theories of practice. London : New Heaven and London Yale

Vega, J. (2013) «Imagining the City : The Difference that Art Makes », In Contemporary Culture, New Directions in Art and Humanities Research, Amsterdam : Amsterdam University Press, pp. 51-61.

Wilkie, F (2004) Out of Place: The Negotiation of Space in Site-Specific Performance

Doctoral thesis, Surrey : University of Surrey

\section{NOTES}

1. Kwon, M (2002) One Place after another, Site-specific art and locational identity, Massachusetts: Massachusetts Institute of Technology, p. 11-31

2. Op. Cit p 26

3. Ibidem p. 160

4. Ce phénomène a été largement abordé par Gustave Pessard, Statuomanie Parisienne - étude critique sur l'abus des statues, Paris, 1911. Maurice Agullion s'est aussi penché sur les conditions de production, les rituels et les emplacements des statues sous la IIIe République tandis que Christel Sniter relate le succès et les échecs de la production parisienne : " 150 statues dédiées à des personnages célèbres y sont érigées contre 26 entre 1815 et 1870. La floraison de statues de Grands hommes répondait à un triple enjeu, esthétique, politique et social » In Les femmes célèbres sont-elles des grands hommes comme les autres? Contribution à l'étude de la statuomanie parisienne, p. 51-72.

5. Comme le note Baker, des écrivains tels que Desnos réfutaient la polémique bourgeoise désignant cette mode comme préjudiciable à l'esthétique parisienne.

6. Plusieurs facteurs ont contribué au déclin des statues telles que le développement de nouvelles formes d'art public et des manifestations anti-monuments.

7. Le projet de recherche s'intitule «L'art public dans le cadre des relations culturelles portugobrésiliennes. ». Voir : http://artepublica.ulusofona.pt/ consulté le 3/05/16

\section{ABSTRACTS}

Recently, mural interventions have been elected as one of the outputs of street artist in various cities of the world. While the sort of composition, contours, colours, and techniques employed in the murals easily make the artist recognizable within the global infrastructure space, some of them embody, highlight or appropriate aspects of the place, concerning many degrees of site specificity. Linked to a research project that concerns public art in the frame of the luso-brazilian cultural relationship, this paper offers an interpretation of how site-specific aspects are presented in the works of Os Gémeos, Vhils, Nunca, and Bicicleta sem Freio. It also aims to discuss questions regarding the concept of site-specificity within street art murals. Although street art may be argued as having heterogeneous artist with different backgrounds raging from graffiti to fine arts that present work in galleries, favorable urban policies have open the public space to legitimize street art work outside these institutional spaces, enabling the resurgence of mural interventions as one of the trends among public art works. 
Les œuvres murales constituent depuis peu l'une des formes de production privilégiées par les street artists dans différentes villes à travers le monde. Si les paramètres de composition, de dessin, de couleur et de technique employés pour les fresques permettent d'identifier aisément leurs auteurs sur les réseaux mondiaux, certains aspects mettent en lumière ou co-agissent avec les spécificités du lieu à différents degrés. Issue du projet de recherche pour l'art public dans le cadre des relations culturelles entre le Portugal et le Brésil, cette étude propose une interprétation des caractéristiques des œuvres d'Os Gémeos, de Vhils, de Nunca et de Bicicleta sem Freio. L'un des objectifs est également le questionnement du concept d'art in situ pour les œuvres murales de street art tout en prenant en compte la nature à la fois locale et mondiale des travaux. Si l'on peut affirmer que le street art - représenté par des artistes aux pratiques très diverses allant du graffiti aux Beaux-Arts et exposé dans différents lieux dont des galeries d'art est hétérogène, les politiques urbaines favorables à son développement ont accordé une nouvelle légitimité aux œuvres de street art en dehors des espaces institutionnels. De telles initiatives ont ainsi permis la résurgence des interventions murales comme l'une des principales pratiques d'œuvres d'art publiques.

\section{INDEX}

Chronological index: XXe siècle, XXIe siècle

Keywords: urban mural, site-specific, street art, public space, avant-garde art practices

Mots-clés: fresque murale, street art, œuvre in situ, espace public, pratiques modernistes, avant-garde

Geographical index: Monde, Portugal, Brésil

\section{AUTHOR}

\section{HELENA ELIAS ET INÊS MARQUES}

Professeurs et Docteurs en Art Publique à l'École de Communication, Architecture, Arts et Technologies, Université Lusophone, Lisbonne, Portugal (ECATI) / ULHT, CICANT 